\title{
Role of Evaluating MGMT Status and 1p36 Deletion in Radiosurgery-Induced Anaplastic Ependymoma That Rapidly and Completely Resolved by Temozolomide Alone: Case Report and Review of the Literature
}

Address for correspondence Seiichiro Hirono, MD, PhD, Department of Neurological Surgery, Chiba University Graduate School of Medicine, 1-8-1 Inohana, Chuoku, Chiba 260-8670, Japan (e-mail: s-hirono@umin.ac.jp).

\author{
Seiichiro Hirono ${ }^{1}$ Yasuo Iwadate ${ }^{1}$ \\ Yukio Nakatani ${ }^{2}$ Naokatsu Saeki ${ }^{1}$

\footnotetext{
${ }^{1}$ Department of Neurological Surgery, Chiba University Graduate School of Medicine, Chuoku, Chiba, Japan

2 Department of Diagnostic Pathology, Chiba University Graduate School of Medicine, Chuoku, Chiba, Japan

${ }^{3}$ Department of Biochemistry and Genetics, Chiba University Graduate School of Medicine, Chuoku, Chiba, Japan
}

Takaki Hiwasa $^{3}$ Masaki Takiguchi ${ }^{3}$

J Neurol Surg Rep 2015;76:e43-e47.

\begin{abstract}
Keywords

- anaplastic ependymoma

- radiosurgery

- MGMT

- 1p36

- temozolomide
\end{abstract}

\section{Introduction}

Stereotactic gamma knife surgery (GKS) is one of the most important treatment modalities for a variety of neurologic diseases including arteriovenous malformation (AVM), benign tumors (acoustic neurinomas, meningiomas, pituitary adenomas, etc.), metastatic tumors, and other conditions (trigeminal neuralgia). Although the risk of tumor induction after conventional radiotherapy is well known, a GKS-induced brain tumor is extremely rare, and only eight cases have been reported to date. ${ }^{1-7}$ Furthermore, no ependymal tumors induced by GKS have been reported, meaning their received

August 11, 2014

accepted

September 20, 2014

published online

January 16, 2015
DOI http://dx.doi.org/

10.1055/s-0034-1396657. ISSN 2193-6366. (c) 2015 Georg Thieme Verlag KG
Stuttgart · New York

License terms

c) $(1) \$$ 
clinical, pathologic, and genetic features are poorly understood.

Anaplastic ependymomas (AEs) are one of the malignant glial tumors histologically characterized by high mitotic activity, microvascular proliferation, and pseudopalisading necrosis. Although the surgical resection is the standard initial strategy, gross total resection can rarely be achieved, and its progressive nature often requires adjuvant radiotherapy and chemotherapy. Although radiotherapy for $\mathrm{AE}$ is widely accepted as effective, reirradiation for a radiationinduced ependymal tumor is unfavorable due to the potential danger of neurotoxic damage. In such cases, chemotherapy is virtually the only treatment option, although chemosensitivity is generally limited with ependymal tumors.

We report our findings in the first case of $\mathrm{AE}$ presented more than 19 years after GKS for a cerebellar AVM. Although the tumor was aggressive enough to disseminate in the ventricle at the time of diagnosis, administration of temozolomide (TMZ) achieved a complete resolution of the postoperative residual tumor in only 5 days. We provide a detailed description of the case as well as a genomic evaluation.

\section{Clinical Summary}

Our patient initially presented with a cerebellar hemorrhage at 57 years of age. A cerebral angiogram demonstrated an
AVM nidus $12 \times 25 \times 23 \mathrm{~mm}$ located mainly in the upper vermis with feeding vessels from the left posterior inferior cerebellar artery and drainage into the transverse sinus. Twelve weeks later, the patient underwent stereotactic radiosurgery (SRS) using GKS. The maximum dose to the nidus was $41.7 \mathrm{~Gy}$, and the prescribed dose at the $60 \%$ isodose line was 25 Gy. The angiogram performed 2 years after GKS confirmed the complete occlusion of the AVM. Routine follow-ups were continued and magnetic resonance (MR) images obtained 16 years later after the GKS demonstrated a cyst formation in the left cerebellar hemisphere. Because the lesion was asymptomatic, the patient was monitored closely using annual MR imaging.

\section{First Surgery and Pathologic Findings}

At 18.5 years after GKS, the patient developed gait unsteadiness and speech difficulty. MR images revealed a partially enhanced 3.3-cm lesion in the left cerebellum (-Fig. 1A). A left suboccipital craniectomy was performed, and the lesion was totally removed (-Fig. 1B). The histologic examination of the first surgical specimen was found to be composed of hematoma and dilated vascular structure surrounded by endothelial cells (-Fig. 1C). These endothelial cells were positive for CD31 ( - Fig. 1D), and the vascular structure lacked elastic fiber. There was no normal cerebellar tissue between the interstices of these capillaries, confirming the
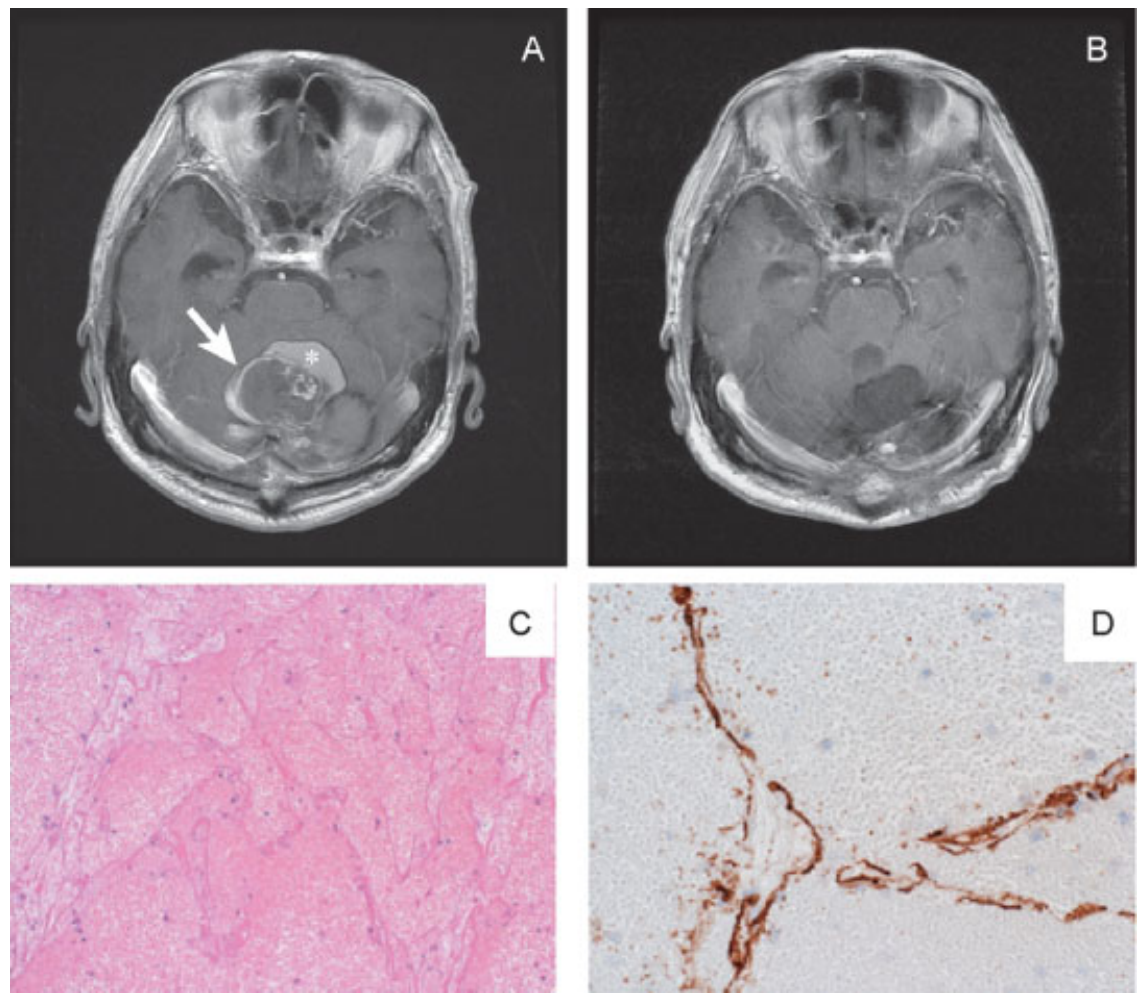

Fig. 1 Axial T1-weighted magnetic resonance (MR) images with gadolinium (A) before and (B) after the first surgery, and (C, D) the pathologic findings. (A) The lesion (arrow) showing partial enhancement surrounded by hematoma (asterisk). (B) MR image showing the total removal of the lesion. (C, D) Pathology specimen of the first surgery. (C) Hematoxylin and eosin staining revealed a fresh hematoma-like lesion with a vague vascular structure covered by CD31-positive endothelial cells (D). 
diagnosis of cavernous malformation. His symptoms rapidly disappeared.

\section{Second Surgery and Pathologic Findings}

At 6 months after the first surgery (19 years after GKS), the patient again developed gait disturbance and cerebellar ataxia of the left side. MR images demonstrated a new enhancing mass in the first surgical cavity ( $\mathbf{F i g}$. 2A). Signs of intraventricular dissemination were also noticed in the posterior horn of the bilateral lateral ventricles ( $\mathbf{- F i g}$. 2B). A subtotal resection of the enhancing lesion was performed ( $\mathbf{F i g} . \mathbf{2 C}$ ), and the pathologic examination revealed the presence of an epithelial arrangement of poorly differentiated tumor cells with marked cellularity, mitotic activity, and irregular shaped nuclei ( - Fig. 3A). Perivascular pseudorosettes and clear cell morphology were also observed (-Fig. 3B). Strong immunostaining for glial fibrillary acidic protein was shown ( - Fig. 3C) and dot-like epithelial membrane antigen positivity. The MIB1 index was $40 \%$ (- Fig. 3D), confirming the diagnosis of $\mathrm{AE}$.

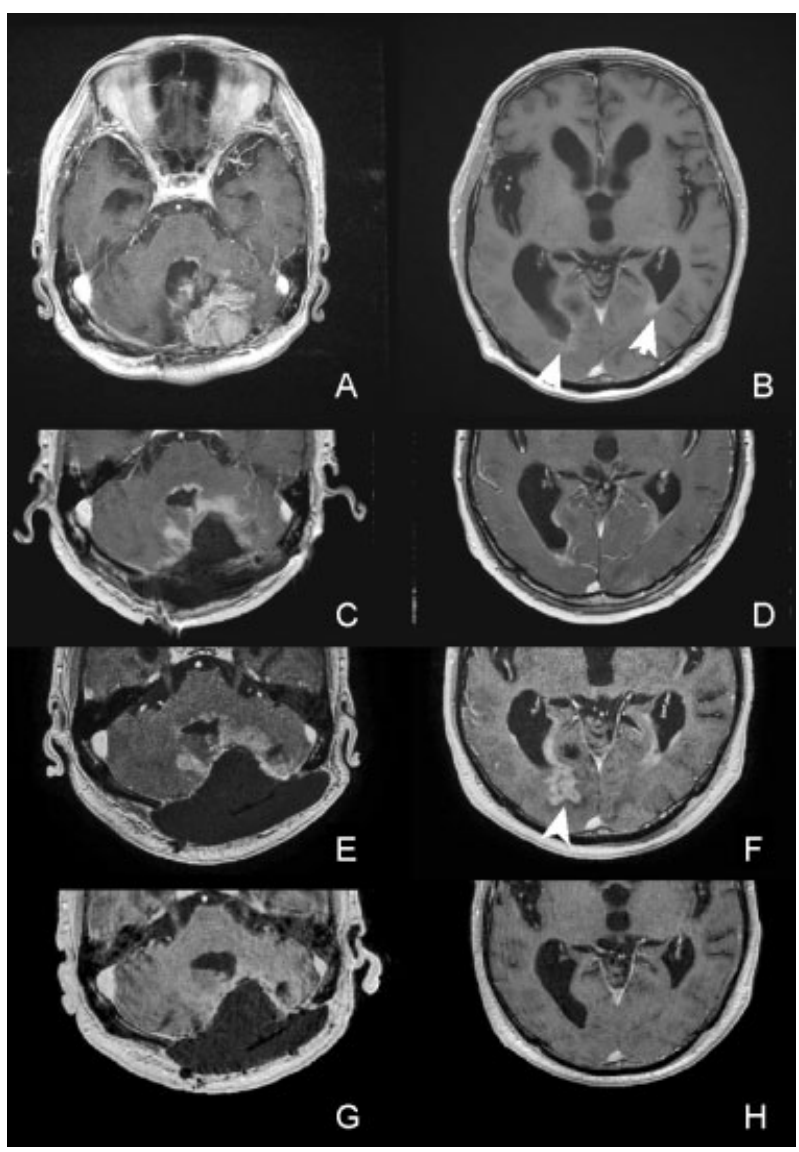

Fig. 2 Axial T1-weighted magnetic resonance (MR) images with gadolinium. (A, B) Nineteen years and 6 months after radiosurgery, an enhancing mass with dissemination in the bilateral posterior horn of the lateral ventricle (arrows). (C, D) MR images after the second surgery showing a residual tumor in the cerebellum. (E, F) Rapid progression of the residual tumor especially in the posterior horn (arrowhead); note the interval between (E, F) and (C, D) was only 18 days. $(G, H)$ Complete resolution after only a 5-day administration of temozolomide (TMZ); note the interval between TMZ initiation and ( $G$, H) was only 16 days.
While waiting for adjuvant chemotherapy, the residual tumor exhibited a remarkable rapid progression ( - Fig. $\mathbf{2 E}, \mathbf{F}$ ) in only 18 days when the postoperative MR images were compared (-Fig. 2C, D). Methylation-specific polymerase chain reaction (MS-PCR) revealed that the promoter region of the $0^{6}$-methylguanine-DNA methyltransferase (MGMT) was methylated. In addition, fluorescence in situ hybridization showed a deletion at chromosome 1p36 (frequency: 97\% of cells). Isocitrate dehydrogenase 1 mutation was not detected immunohistochemically. Taken together, the patient was treated adjuvantly with TMZ using the Stupp et al regimen for recurrent glioblastoma (GBM), whereby TMZ (150-200 $\mathrm{mg} / \mathrm{m}^{2} /$ day for 5 days) was administered monthly. Surprisingly, MR images obtained only 16 days after the induction of TMZ demonstrated a complete response (CR) of the residual tumor ( - Fig. 2G, H). MR images after three cycles of TMZ still showed the $\mathrm{CR}$ status.

\section{Discussion}

Cahan et $\mathrm{al}^{8}$ established the following criteria for a radiationinduced neoplasm: The tumor must appear within the irradiated field, the tumor must not be present before irradiation, a sufficient latency period must elapse between the irradiation and the appearance of the tumor, the histology must be distinct from the original irradiated lesion, and the patient should not have a genetic predisposition to a second malignancy. Our case fulfills all these criteria, in both the cavernous hemangioma and the AE. Because the first craniectomy achieved the total removal of the cavernous malformation, we believed these two tumors were independently induced in the same patient by the GKS almost 2 decades earlier. It is also interesting that the $\mathrm{AE}$ in this patient developed within only 6 months after the first microsurgery.

There have been only three case reports of conventional radiation-induced intracranial ependymal tumor. ${ }^{9-11}$ In addition, an SRS-induced brain tumor is extremely rare. To the best of our knowledge, there have been only eight case reports $^{1-7}$ : four cases of GBM, two cases of meningioma, and two cases of anaplastic astrocytoma, with the average interval between GKS and the tumorigenesis of 9.5 years (range: 5.2-19 years). Thus our case report is the first report of an intracranial ependymal tumor induced by GKS. SRS has the advantage of a rapid radiation falloff outside the target volume, which makes the surrounding tissues receive a much lower radiation dose. No increased risk of malignancy was observed in a retrospective cohort study of $\sim 5,000$ GKStreated patients with an average follow-up period of 6.1 years. ${ }^{12}$ However, our case of tumor induction more than 19 years after GKS reminds us again that long-term followups are essential for those treated by GKS because more potential secondary tumors will be observed as a greater interval of time passes.

In retrospect, no components suggesting ependymal tumor were detected in the first surgical specimen. Most of the cells surrounded by the endothelial cells in the first surgical specimen were red blood cells, and no perivascular pseudorosettes were observed. Although microvascular 

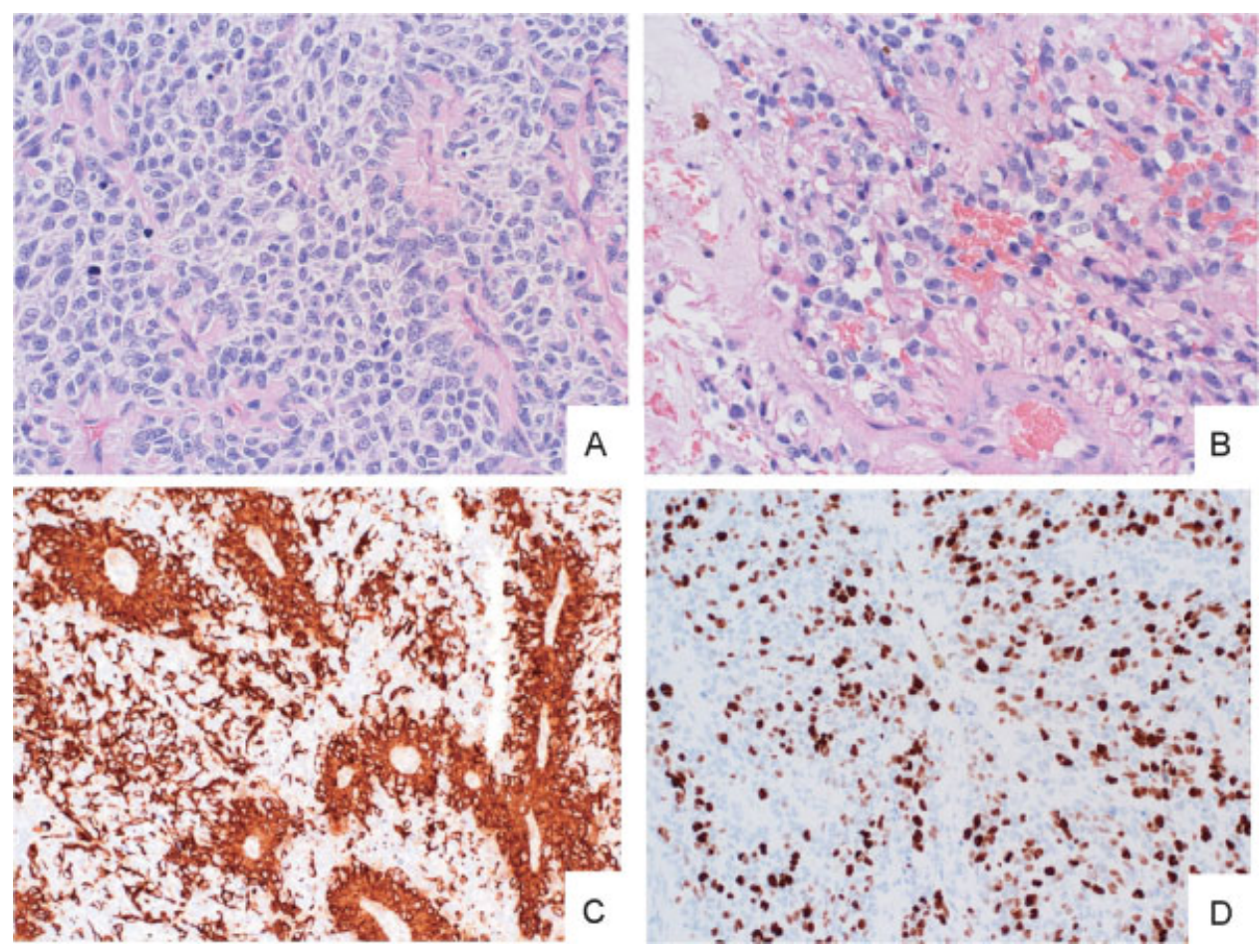

Fig. 3 Pathology specimen of the second surgery. Hematoxylin and eosin staining revealed (A) the epithelial arrangement of the poorly differentiated tumor cells with marked cellularity and mitotic activity. (B) Perivascular pseudorosettes were also observed. These are histologic hallmarks of an anaplastic ependymoma. Tumor cells were immunoreactive for glial fibrillary acidic protein (C), and the MIB-1 index was $40 \%$ (D).

proliferation was observed in the second ependymal tumor, those vascular structures are a common hallmark of ependymal tumors, and no clear evidence to diagnose the concomitant development of $\mathrm{AE}$ with cavernous malformation was found. Therefore, it is reasonable to assume that the $\mathrm{AE}$ developed independently shortly after the total removal of the cavernous malformation. Regarding the carcinogenesis of the $\mathrm{AE}$, it is interesting that this tumor developed within only 6 months after the first surgery, which well reflects the high MIB-1 index of $40 \%$.

Whereas radiotherapy is recognized as an effective treatment option for $\mathrm{AE}$, reirradiation for a radiation-induced ependymal tumor is unfavorable due to the potential risk of neurotoxicity. Therefore, adjuvant chemotherapy was the only remaining choice in our case. However, no clear efficacy of any regimen including TMZ has been demonstrated in cases of ependymal tumors. In contrast, the methylated promoter region of MGMT has been proved to be a prognostic factor for patients with GBM treated with TMZ, ${ }^{13}$ but no similar evaluation has been performed in patients with ependymal tumors.

Koos et al ${ }^{14}$ reported the interesting finding that MGMT promoter methylation occurred significantly less frequently in subjects with primary ependymal tumors compared with those with malignant gliomas in a malignancy grade-dependent manner, which means MGMT methylation is less highly anticipated in AEs. Thus a chemotherapy regimen including TMZ would not seem to be beneficial for patients with primary ependymal tumors. However, we analyzed the MGMT promoter status with MS-PCR and revealed that it was methylated, suggesting the possible benefit of TMZ.
There is another case report of AE induced by conventional radiation in childhood with a methylated MGMT promoter that was evaluated immunohistochemically. ${ }^{11}$ This case also demonstrated a similar remarkable response to TMZ. This suggests that radiation-induced ependymal tumors may have a different genetic background and result from different mechanisms of carcinogenesis. Therefore, even in ependymal tumors, especially when radiation induced, genetic evaluations of MGMT promoter status should be performed to select those patients who might benefit from TMZ.

The combined deletion of $1 \mathrm{p} 36$ and $19 \mathrm{q} 13$ was shown to predict better chemosensitivity and prognosis in cases of anaplastic oligodendroglioma, ${ }^{15}$ but insufficient evaluations have been performed in cases of ependymal tumors. According to a small study of only 28 patients with ependymoma, 1 p36 loss was observed in only $29 \%$ of the cases. ${ }^{16}$ Our patients also exhibited deletion of $1 \mathrm{p} 36$, which may be involved in the dramatic clinical and radiologic improvement by TMZ, but this also must be validated in much larger patient populations.

\section{Conclusion}

We present a case of GKS-induced AE with a methylated MGMT promoter and deletion of $1 \mathrm{p} 36$ that developed 19 years after GKS. This report reemphasizes the need for many longterm follow-ups, especially in GKS-treated patients. The evaluation of not only MGMT promoter status but also the chromosomal deletion of $1 \mathrm{p} 36$ is useful for the potential benefit of TMZ even in ependymal tumors. A GKS-induced tumor may have a different genetic background compared 
with the primary tumor, which results from the different pathogenesis of the tumor and may involve chemosensitivity.

\section{References}

1 Yu JS, Yong WH, Wilson D, Black KL. Glioblastoma induction after radiosurgery for meningioma. Lancet 2000;356(9241):1576-1577

2 Kaido T, Hoshida T, Uranishi R, et al. Radiosurgery-induced brain tumor. Case report. J Neurosurg 2001;95(4):710-713

3 Shamisa A, Bance M, Nag S, et al. Glioblastoma multiforme occurring in a patient treated with gamma knife surgery. Case report and review of the literature. J Neurosurg 2001;94(5):816-821

4 McIver JI, Pollock BE. Radiation-induced tumor after stereotactic radiosurgery and whole brain radiotherapy: case report and literature review. J Neurooncol 2004;66(3):301-305

5 Sheehan J, Yen CP, Steiner L. Gamma knife surgery-induced meningioma. Report of two cases and review of the literature. J Neurosurg 2006;105(2):325-329

6 Berman EL, Eade TN, Brown D, et al. Radiation-induced tumor after stereotactic radiosurgery for an arteriovenous malformation: case report. Neurosurgery 2007;61(5):E1099; discussion E1099

7 Starke RM, Yen CP, Chen CJ, et al. An updated assessment of the risk of radiation-induced neoplasia after radiosurgery of arteriovenous malformations. World Neurosurg 2014;82(3-4):395-401

8 Cahan WG, Woodard HQ Higinbotham NL, Steward FW, Coley BL. Sarcoma arising in irradiated bone: report of eleven cases. 1948. Cancer 1998;82(1):8-34
9 Haselow RE, Nesbit M, Dehner LP, Khan FM, McHugh R, Levitt SH. Second neoplasms following megavoltage radiation in a pediatric population. Cancer 1978;42(3):1185-1191

10 Anderson JR, Treip CS. Radiation-induced intracranial neoplasms. A report of three possible cases. Cancer 1984;53(3): 426-429

11 Khoo HM, Kishima H, Kinoshita M, et al. Radiation-induced anaplastic ependymoma with a remarkable clinical response to temozolomide: a case report. Br J Neurosurg 2013;27(2): 259-261

12 Rowe J, Grainger A, Walton L, Silcocks P, Radatz M, Kemeny A. Risk of malignancy after gamma knife stereotactic radiosurgery. Neurosurgery 2007;60(1):60-65; discussion 65-66

13 Hegi ME, Diserens AC, Gorlia T, et al. MGMT gene silencing and benefit from temozolomide in glioblastoma. N Engl J Med 2005; 352(10):997-1003

14 Koos B, Peetz-Dienhart S, Riesmeier B, Frühwald MC, Hasselblatt M. O(6)-methylguanine-DNA methyltransferase (MGMT) promoter methylation is significantly less frequent in ependymal tumours as compared to malignant astrocytic gliomas. Neuropathol Appl Neurobiol 2010;36(4):356-358

15 Alentorn A, Sanson M, Idbaih A. Oligodendrogliomas: new insights from the genetics and perspectives. Curr Opin Oncol 2012;24(6): 687-693

16 Zheng PP, Pang JC, Hui AB, Ng HK. Comparative genomic hybridization detects losses of chromosomes 22 and 16 as the most common recurrent genetic alterations in primary ependymomas. Cancer Genet Cytogenet 2000;122(1):18-25 